Math. Model. Nat. Phenom.

Vol. 4, No. 6, 2009, pp. 135-155

DOI: $10.1051 / \mathrm{mmnp} / 20094605$

\title{
Seasonality, Climate Cycles and Body Size Evolution
}

\author{
T. A. Troost ${ }^{1 *}$, J. A. van $\operatorname{Dam}^{2,3}$, B. W. Kooi ${ }^{4}$ and E. Tuenter \\ ${ }^{1}$ Deltares, Department of Ecosystem Analysis and Assessment \\ Rotterdamseweg 185, 2600 MH Delft, The Netherlands \\ ${ }^{2}$ Faculty of Earth and Life Sciences, Dept. of Paleoclimatology and Geomorphology \\ Vrije Universiteit, De Boelelaan 1085, 1081 HV Amsterdam, The Netherlands \\ ${ }^{3}$ Institut Catala de Paleontologia (ICP) \\ Campus de la UAB, Modul ICP, E-08193 Cerdanyola del Valles, Spain \\ ${ }^{4}$ Faculty of Earth and Life Sciences, Dept. of Theoretical Biology \\ Vrije Universiteit, De Boelelaan 1085, 1081 HV Amsterdam, The Netherlands \\ ${ }^{5}$ Institute for Marine and Atmospheric research Utrecht (IMAU) \\ Princetonplein 5, 3584 CC Utrecht, The Netherlands
}

\begin{abstract}
The seasonality hypothesis states that climates characterized by large annual cycles select for large body sizes. In order to study the effects of seasonality on the evolution of body size, we use a model that is based on physiological rules and first principles. At the ecological time scale, our model results show that both larger productivity and seasonality may lead to larger body sizes. Our model is the first dynamic and process-based model to support the seasonality hypothesis and hence demonstrates the importance of basing models on physiological processes. We focus not only on variability at the ecological time scale, but also on the temporal variations in seasonality existing at geological time scales. A particularly strong forcing of seasonality exists on the scale of 20,000-400,000 years, the scale of Milankovitch cycles. Therefore, we simulated the evolutionary response of body size to a Milankovitch-type of forcing of climate and food density. Results illustrate that for a given level of investment in reserves body size may track climatic cycles, and that below a certain seasonality threshold the body size will decrease rapidly, leading to extinction.
\end{abstract}

Key words: adaptive dynamics, dynamic energy budget, Milankovitch cycles, seasonality hypothesis

AMS subject classification: 86A60, 92D15, 92D25

*Corresponding author. E-mail: tineke.troost@deltares.nl 


\section{Introduction}

Lindstedt and Boyce (1985) stated their 'seasonality hypothesis' saying that seasonal environments select for large body sizes $[2,3]$. The underlying mechanism is thought to be related to starvation, as larger organisms have larger energy reserves that will last longer under starvation conditions $[26,36]$.

The seasonality hypothesis has been tested against field data by searching for geographical gradients in body size that correlate with gradients in seasonality, but the results are nondecisive. Positive relations between body size and seasonality were found for muskrats [2], western bobcats [44], western rattlesnake [1], weevils [4] and sifakas [23]. In contrast, data on moose [10] and thirteen species of western Palaearctic carnivores [29] did not show clear correlations and thus did not support the hypothesis. The variability of the results may be explained by the fact that body size is not only related to starvation, but also to a range of other physiological processes such as ingestion, maintenance, reproduction and thermo-regulation. As was previously pointed out by Dunsbrack and Ramsay (1993), these (inter)relationships and processes may cloud the effects of seasonality on body size.

Mathematical models may help to study the many interrelationships between body size and physiological processes. In their paper on the seasonality hypothesis, Lindstedt and Boyce (1985) already provided a model to support the hypothesis. However, their model is static and based on allometric relationships, thus providing a mainly descriptive relation between body size, energy reserves, and fasting endurance. Various dynamic and more process-based type of models have been developed to better understand the effect of seasonality on a variety of life history parameters and storage dynamics. Cohen and Parnas (1976) used a model to study the relationship between variability and storage level, and optimised allocation patterns. They found that variability leads to higher levels of storage, but they made no predictions on body size. Boyce (1979) studied the relation between seasonality and life history parameters ( $\mathrm{r}$ - versus K-selection), and his results can be interpreted as being consistent with the seasonality hypothesis. Contradictory, Shertzer and Ellner (2002) found that variable environments select for smaller body sizes. Clearly, their result does not support the hypothesis.

The present paper aims to study again the effects of seasonality on the evolution of body size by means of a modeling approach. Our model differs from the previously mentioned models because it is fully based on physiological rules for uptake and use of energy and material; the dynamics of biomass and reserves are derived from first principles such as obeying mass balance, and tested against a large amount of experimental data, which modeling framework is referred to as Dynamic Energy Budget (DEB) theory [21]. A relationship between body size and energy reserves is not included explicitly, but follows from the assumptions underlying the dynamics of the energy-reserve. To test our model, we study the body size response of our model organisms at different seasonalities. For this, we examine the model's evolutionary equilibria with use of Adaptive Dynamics (AD) theory.

Environmental variability does not only exist at the annual time scale, but also at geological time scales. For instance, the seasonal amplitude periodically increases and decreases 
over time. A particularly strong forcing of seasonality occurs on the scale of 20,000-400,000 years, the scale of Milankovitch cycles [32, 22], which have been extensively documented in the geological record $[15,34,16]$. Solar radiation reaching the earth's atmosphere is a function of three orbital parameters: the wobbling of the earth's axis (precession, mean period 21,000 years), the tilt of the axis (obliquity, 41,000 year period), and the shape of the earth orbit around the sun (eccentricity, main periods 100,000 and 400,000 years). The effect of precession is that the distance from earth to sun varies for each of the seasons. The climatic effects of precession on seasonality are present on all latitudes, with periods of high seasonality alternating with periods of low seasonality. Obliquity basically creates the seasons, with the strongest effects on high latitudes. The direct contribution of eccentricity to insolation is low, but because eccentricity modulates precession, 100,000 and 400,000 year periodicities are also well recorded in the geological record.

Like seasonality, also body sizes within fossil lineages are known to vary strongly at a geological time scale. To study whether fluctuations in body size at such time scales can be attributed to periodic variations in seasonality, we test the body size response of our model organisms to a Milankovitch-type of climate forcing. For this we study the evolutionary trajectories resulting from a stochastic simulation process.

The population dynamics of rodents are well known, and often driven by periodic environmental factors such as rainfall $[37,6,25,28,40]$. Also, evidence for cyclical patterns in body size has been found in the fossil record of rodents [39]. Therefore, we have based our model on these animals. Of course, the precession cycles and rodents only act as an example to show the effect of large scale climatic fluctuations on evolution of body size, and the model is equally applicable to other climate cycles as well as to other organisms.

\section{Methods}

\section{1. $\quad$ Model description}

We use a model that is based on a model for rotifers developed in [18, 19], and adjusted it to account for rodents living in an area without migration from the surrounding area and with food entering the system with a periodically varying density. The individual rodents can be described by their body size (length) and their reserves (energy density). The energy density is scaled by the maximum energy density $\left[E_{\mathrm{m}}\right]$, so that the resulting scaled energy density $e_{1}=\left[E_{1}\right] /\left[E_{\mathrm{m}}\right]$ is dimensionless and may range between 0 to 1 . As rodents quickly grow into their adult size, the model ignores the juvenile stage. Neither does it consider an explicit embryonic stage. All individuals have the same body size $\ell_{\mathrm{A}}$ as well as the same energy density $e_{1}$. As a result, the rodent population is unstructured, and can be described by two state variables, the population's 'structural biomass' (the total amount of biovolume per hectare $X_{1}$ ) and its 'reserve density' (energy per structural volume $\left[E_{1}\right]=E_{1} / X_{1}$ ). Note that square brackets [ ] around a variable imply that this variable is expressed per volume. 
The resulting model is formed by the following ordinary differential equations (ODEs):

$$
\begin{aligned}
\frac{\mathrm{d} X_{0}}{\mathrm{~d} t} & =\left(X_{0, \text { in }}(t)-X_{0}\right) D-f\left(X_{0}\right)\left\{I_{\mathrm{m}}\right\} \frac{X_{1}}{\ell_{\mathrm{A}}}, \\
\frac{\mathrm{d} e_{1}}{\mathrm{~d} t} & =\frac{v}{\ell_{\mathrm{A}}}\left(f\left(X_{0}\right)-e_{1}\right), \\
\frac{\mathrm{d} X_{1}}{\mathrm{~d} t} & =\left(R\left(e_{1}\right)-h\right) X_{1},
\end{aligned}
$$

where $X_{0}$ is the food density in the system, and $X_{0, \text { in }}(t)$ is the food density that enters the system at rate $D$; its periodic forcing is discussed in at the end of this section. $X_{1} / \ell_{\mathrm{A}}$ is the total rodent surface-area per hectare, and $\left\{I_{\mathrm{m}}\right\}$ is the surface-area-specific maximum ingestion rate. Note that the curly brackets \{\} imply that the variable is expressed per surface area. The function $f\left(X_{0}\right)$ is their functional response, which is assumed to be of Holling type-II ,

$$
f\left(X_{0}\right)=\frac{X_{0}}{K+X_{0}}
$$

where $K$ is the saturation constant. Parameter $v$ is the energy conductance and $h$ the rodent per capita mortality rate which can be due to natural death or a constant predation. Since individuals do not grow, all consumed food is used for maintenance and reproduction. The reproduction rate $R\left(e_{1}\right)$ is given by

$$
R\left(e_{1}\right)=\frac{v / \ell_{\mathrm{A}} e_{1}-k_{\mathrm{M}} g}{g+\frac{3}{4} g k_{\mathrm{M}} \ell_{\mathrm{A}} / v+e_{1}} .
$$

This expression has been derived in $[19,18]$. It is the ratio between the rate with which energy becomes available for reproduction and the amount of energy needed per embryo. The rate at which the energy is liberated from the energy reserves equals the conductance $v / \ell_{\mathrm{A}}$ times the scaled energy density $e_{1}$ of the mother. From this mobilised energy, the costs for maintenance have to be paid. These costs are equal to the maintenance rate $k_{\mathrm{M}}$ times the energy investment ratio $g$, the cost building new structural biomass. The denominator of Eqn. (2.3) is the (scaled) energy density required to produce embryo's. The costs for a newborn individual equals the cost building new structural biomass $g$, the cost for maintenance during the embryonic period, $\frac{3}{4} g k_{\mathrm{M}} \ell_{\mathrm{A}} / v[19,18]$ plus the energy density of a newborn individual, $e_{1}$.

Parameter values for physiological processes were based on average values for rodent species found in literature. Weights were converted to biovolumes using a volume-specific density of $1 \mathrm{~g} \mathrm{~cm}^{-3}$. The average incoming food density, termed productivity since the dilution rate of food $D$ is constant, $\bar{X}_{0, \text { in }}$, was set to 100 or $10 \mathrm{~kg} \mathrm{ha}^{-1}$, as to result in realistic rodent densities. The saturation constant $K$ was chosen as one-tenth of the maximum possible food density, such that the scaled functional response $f$ could range from 0 to a value close to 1 . For a more detailed explanation of the model, including derivations of $R$, 
Table 1: Used symbols; $\mathrm{t}=$ time, $\mathrm{L}=$ length of individual, $\mathrm{l}=$ length of area, $\mathrm{m}=$ mass, $\mathrm{e}=$ energy. Note that square brackets around a variable indicate that this variable is expressed per volume, while curly brackets indicate that it is expressed per surface area

\begin{tabular}{lll}
\hline Symbol & Dimension & Interpretation \\
$D$ & $\mathrm{t}^{-1}$ & Dilution rate of food \\
{$[E],\left[E_{\mathrm{m}}\right]$} & $\mathrm{e} \mathrm{L}^{-3}$ & Energy density, and maximum (storage capacity) \\
$f$ & - & Scaled functional response \\
$g$ & - & Energy investment ratio: $g=\alpha v /\left(k_{\mathrm{M}} \ell_{\mathrm{A}}\right)$ \\
$h$ & $\mathrm{t}^{-1}$ & Per capita mortality rate \\
$\left\{I_{\mathrm{m}}\right\}$ & $\mathrm{L}^{3} \mathrm{~L}^{-2} \mathrm{t}^{-1}$ & Surface-area-specific maximum intake rate: $\left\{I_{\mathrm{m}}\right\}=\ell_{\mathrm{A}}\left[I_{\mathrm{m}}\right]$ \\
{$\left[I_{\mathrm{m}}\right]$} & $\mathrm{L}^{3} \mathrm{~L}^{-3} \mathrm{t}^{-1}$ & Volume-specific maximum intake rate. \\
$k_{\mathrm{M}}$ & $\mathrm{t}^{-1}$ & Maintenance rate coefficient \\
$R$ & $\mathrm{t}^{-1}$ & Reproduction rate \\
$T_{0}, T_{\mathrm{p}}$ & $\mathrm{t}$ & Period length of seasonal cycle, and of precession cycle \\
$X_{0, \text { in }}(\mathrm{t}), \bar{X}_{0, \text { in }}$ & $\mathrm{L}^{3} \mathrm{l}^{-2}$ & Incoming resource density, function and average \\
$\alpha$ & - & Fraction of the asymptotic size that is reached \\
$\epsilon, \epsilon_{\mathrm{p}}$ & - & Amplitude of the seasonal cycle, and of the precession cycle \\
$v$ & $\mathrm{~L} \mathrm{t}^{-1}$ & Energy conductance \\
State Variables & & \\
$X_{1}, X_{0}$ & $\mathrm{~L}^{3} \mathrm{l}^{-2}$ & Rodent and resource biovolume density \\
$e_{1}$ & - & Scaled energy density \\
Adaptive traits & & \\
$\ell_{\mathrm{A}}$ & & $\mathrm{L}$ \\
$\ell_{\mathrm{m}}$ & & Adult rodent length \\
& & Maximum rodent length $\ell_{\mathrm{m}}=\ell_{\mathrm{A}} / \alpha$ \\
& &
\end{tabular}

readers may want to consult $[19,18]$. Parameters and variables of the model are summarised in Table 1.

The default parameter values are listed in Table 2 .

During the winter, the rodents may deplete their energy storage. In case they can no longer meet their maintenance requirements $\left(k_{\mathrm{M}} g>v / \ell_{\mathrm{A}} e_{1}\right.$, so that $\left.R\left(e_{1}\right)<0\right)$, starvation will start to occur. Note that the reserve density does not need to be zero for such shortages to arise, as it is not only the reserve density but also its mobilisation rate $v / \ell_{\mathrm{A}}$ that may be physiologically limiting.

We also consider the 'no starvation threshold' body size situation in which the body size is fixed by the requirement that during the periodic solution the reproduction rate $R$ is non-negative for the whole period and zero at at least on point in time. In biological terms 
Table 2: Default parameter values. Notice that $g=\alpha v /\left(k_{\mathrm{M}} \ell_{\mathrm{A}}\right)=0.1 / \ell_{\mathrm{A}}$

\begin{tabular}{llll}
\hline Symbol & Value & Unit & Reference (if relevant) \\
$D$ & 0.01 & $\mathrm{~d}^{-1}$ & \\
$h$ & 0.002 & $\mathrm{~d}^{-1}$ & {$[11]$} \\
{$\left[I_{\mathrm{m}}\right]$} & 0.05 & $\mathrm{mg} \mathrm{ml}^{-1} \mathrm{~d}^{-1}$ & {$[43]$} \\
$k_{\mathrm{M}}$ & 0.5 & $\mathrm{~d}^{-1}$ & {$[21]$} \\
$K$ & 1 & $\mathrm{~kg} \mathrm{ha}^{-1}$ & $1 / 10$ of $\bar{X}_{0, \text { in }}$ \\
$T_{0}$ & 365 & $\mathrm{~d}$ & \\
$v$ & 0.2 & $\mathrm{~cm} \mathrm{~d}^{-1}$ & {$[21]$} \\
$\bar{X}_{0, \text { in }}$ & 10 & $\mathrm{~kg} \mathrm{ha}^{-1}$ & \\
$\alpha$ & 0.25 & - & - \\
$\beta_{\mathrm{p}}$ & 1. & - & - \\
\hline
\end{tabular}

this means that during the whole period the reserves are sufficient to maintenance needs.

Scaling considerations. In the present study, body size $\ell_{\mathrm{A}}$ is assumed to be subject to evolution. Therefore, we included various body-size scaling relationships that were not considered in the original DEB model $[19,18]$, where body size was assumed to be fixed. The adult size $\ell_{\mathrm{A}}$ is assumed to lie at a fraction $\alpha$ of the maximum body size $\ell_{\mathrm{m}}$, that is, $\ell_{\mathrm{A}}=\alpha \ell_{\mathrm{m}}$. The maximum body size $\ell_{\mathrm{m}}^{3}$, follows from an expression central to DEB theory, $\ell_{\mathrm{m}}=v /\left(k_{\mathrm{M}} g\right)$ $[21]$.

As a result the physiological parameters $g$, and $\left\{I_{\mathrm{m}}\right\}$ will become proportional to the maximum body size or population body size. Firstly, the energy investment ratio $g$ depends on body volume $\ell_{\mathrm{m}}^{3}$, following

$$
g=\frac{v}{k_{\mathrm{M}} \ell_{\mathrm{m}}}=\frac{\alpha v}{k_{\mathrm{M}} \ell_{\mathrm{A}}} .
$$

Secondly, the surface-area specific ingestion rate is proportional to size

$$
\left\{I_{\mathrm{m}}\right\}=\ell_{\mathrm{A}}\left[I_{\mathrm{m}}\right]
$$

in which $\left[I_{\mathrm{m}}\right]$ is the constant volume-specific ingestion rate, following a primary scaling relationship in DEB theory. We recall that the following physiological parameters are constant: $v, k_{\mathrm{M}}, \alpha$ and $\left[I_{\mathrm{m}}\right]$.

The periodic environment. As primary productivity (and hence food availability for rodents) strongly depends on precipitation [24], the temporal change in seasonality was incorporated in the function for the incoming food density, $X_{0, \text { in }}(t)$. This incoming density has a sinusoidal shape which is specified by the amplitude $\epsilon$, the average incoming food 
density or productivity $\bar{X}_{0, \text { in }}$ and the period length $T_{0}$, such that the density is always nonnegative:

$$
X_{0, \text { in }}(t)=\left\{\begin{array}{ll}
\bar{X}_{0, \text { in }}\left(1+\beta_{\mathrm{p}} \epsilon \sin \left(2 \pi t / T_{0}\right)\right) & , 1+\epsilon \sin \left(2 \pi t / T_{0}\right)>0 \\
0 & , 1+\beta_{\mathrm{p}} \epsilon \sin \left(2 \pi t / T_{0}\right) \leq 0 .
\end{array} .\right.
$$

Note that, strictly speaking, $\bar{X}_{0, \text { in }}$ is not the time average of $X_{0, \text { in }}(t)$ due to its truncation at 0 . The parameter $\beta_{\mathrm{p}}$ allows for the incorporation of the effects of precession on seasonality, which is discussed later in this article. Until then, its value is set to one $\left(\beta_{\mathrm{p}}=1\right)$.

\subsection{Evolutionary equilibria and Pairwise Invasibility Plots}

In this paper, we assume body size to be inherited from parents to offspring, but mutants with a different body size may invade and replace the resident population. A series of such replacements will lead to phenotypic change of the population. To find the evolutionary equilibrium, i.e. the body size to which the organisms will eventually evolve, we use the ADtheory; this theory helps analysing phenotypic evolution under frequency-dependent selection $[30,8,7,13,14,41]$.

The 'canonical equation' of (AD)-theory [8] provides a deterministic approximation of the evolutionary trajectory of body size. For the approximation to hold in periodic (seasonal) systems the mutant invasion must be slow with respect to the dynamics of the periodic

attractor. For the body size of the rodents in our population the canonical equation comes down to:

$$
\frac{\mathrm{d} \ell_{\mathrm{res}}}{\mathrm{d} t}=\left.\frac{1}{2} k \frac{X_{1}}{\ell_{\mathrm{res}}^{3}} \frac{\partial}{\partial \ell_{\mathrm{mut}}} s_{\mathrm{mut}}\right|_{\ell_{\mathrm{mut}}=\ell_{\mathrm{res}}}
$$

where $\ell_{\text {res }}$ and $\ell_{\text {mut }}$ are the trait values $\ell_{\mathrm{A}}$ of the resident and the mutant population, respectively; $k$ is the mutation-rate parameter involving the fraction of mutations per birth and the mean size of the mutational step; $X_{1} / \ell_{\text {res }}^{3}$ is the mean population size (in number of rodents per hectare). The invasion fitness of a mutant $s_{\text {mut }}$ is defined as its long-term per capita growth rate while being rare in the environment, denoted by $E\left(\ell_{\text {res }}\right)$ and set by the resident population at its ecological equilibrium.

In constant environments, the mutant invasion fitness is equal to the net specific growth rate of the mutant, but in seasonal systems, the mutant invasion fitness comes down to the mutant's net growth rate averaged over the environmental cycle period $T_{0}[31,20]$ :

$$
s_{\mathrm{mut}}=T_{0}^{-1} \int_{0}^{T_{0}} R_{\mathrm{mut}}\left(\ell_{\mathrm{mut}}, E\left(t, \ell_{\mathrm{res}}\right)\right) \mathrm{d} t-h .
$$

Eventually, the rodents may reach a body size at which the fitness gradient has vanished:

$$
\left.\frac{\partial}{\partial \ell_{\mathrm{mut}}} s_{\mathrm{mut}}\right|_{\ell_{\mathrm{mut}}=\ell_{\mathrm{res}}}=0
$$


Such a 'singular strategy' is an equilibrium point of the canonical equation (Eqn. (2.7)), and is called an evolutionary equilibrium. Alternatively, the rodent's body size will end at an extreme, in our case zero body size. The evolutionary equilibria can be calculated by application of the bifurcation theory [42]. With bifurcation theory, points in the trait space where invasibility changes correspond to so called transcritical bifurcations. Available standard computer packages can be used for continuation and this yields directly the evolutionary equilibrium of the body size plotted as a function of the seasonality, $\epsilon$.

Related to the evolutionary equilibria is the study of the so-called pairwise invasibility plot (PIP) [12], which yields the dynamic properties of the evolutionary equilibria. These plots can be constructed using the following system:

$$
\begin{aligned}
\frac{\mathrm{d} X_{0}}{\mathrm{~d} t} & =\left(X_{0, \text { in }}(t)-X_{0}\right) D-f\left(X_{0}\right)\left\{I_{\mathrm{m}}\right\} \frac{X_{1 \mathrm{res}}}{\ell_{\text {res }}}-f\left(X_{0}\right)\left\{I_{\mathrm{m}}\right\} \frac{X_{1 \mathrm{mut}}}{\ell_{\text {mut }}}, \\
\frac{\mathrm{d} e_{1 \mathrm{res}}}{\mathrm{d} t} & =\frac{v}{\ell_{\text {res }}}\left(f\left(X_{0}\right)-e_{1 \mathrm{res}}\right) \\
\frac{\mathrm{d} e_{1 \mathrm{mut}}}{\mathrm{d} t} & =\frac{v}{\ell_{\mathrm{mut}}}\left(f\left(X_{0}\right)-e_{1 \mathrm{mut}}\right) \\
\frac{\mathrm{d} X_{1 \mathrm{res}}}{\mathrm{d} t} & =\left(R_{\mathrm{res}}\left(e_{1 \mathrm{res}}\right)-h\right) X_{1 \mathrm{res}}, \\
\frac{\mathrm{d} X_{1 \mathrm{mut}}}{\mathrm{d} t} & =\left(R_{\text {mut }}\left(e_{1 \mathrm{mut}}\right)-h\right) X_{1 \mathrm{mut}}
\end{aligned}
$$

in which a resident and a mutant population compete for food. For a similar population model in a fluctuating environment, the used analysis methods are discussed in more detail in $[20]$.

\subsection{Evolutionary trajectories}

Our study involves three different time-scales. The ecological time-scale at which the state variables of the ecosystem change is fixed by the reproduction rate or the generation time. The second time-scale, the evolutionary time-scale, is associated with the mutation rate, which is the rate with which the evolutionary traits change over time. The third time-scale is related to the geological time scale (Milankovitch cycles).

In case of a constant annual cycle, the ecological and evolutionary time scales are of a different order and it is therefore reasonable to assume that mutant populations with better adapted trait values have replaced the resident population before a next mutation occurs. This means that before each mutation, the system has reached its periodic solution. As a result, these trajectories can be deterministically approximated with use of the canonical equation of adaptive dynamics (Eqn. (2.7)), as is discussed above.

However, the period of geological cycles modulating the annual seasonality is large and typically covers multiple generations. Therefore, evolution takes place on the same timescale as the fluctuations in the seasonality. In this situation the focus comes to lie more with the evolutionary trajectories than with the evolutionary equilibria, as these may not always 
be reached in the fluctuating environment. Also, equilibrium assumptions mentioned above may be violated, in which case AD-theory can no longer be used. To avoid this potential problem, we simulated the stochastic mutation process.

The evolutionary trajectories were calculated by means of stochastic simulations using Eqn. (2.9). Each time a new mutant is introduced, this set of oDEs is extended by a pair of ODEs for the energy reserves and structural biomass. To limit the number of potential lineages, they were grouped in size classes with a width of $\log _{10}(0.005)$.

In our simulations mutations were introduced at fixed time intervals and the mutations were assumed to be normally distributed around the parent maximum body size $\ell_{\mathrm{m}}$ with variance $\sigma=0.02 \mathrm{~cm}^{2}$. The mutation rate $k$ was set to 0.00005 per birth. Mutants were assumed to enter the system with the same reserve density as their parent and, because they were assumed to be rare, with a very low initial biomass density (a numerical value of $10^{-20}$ was used). the threshold for extinction was also set to this very small value.

The body size response of rodents was studied over a period of a few precession cycles, during which the seasonality (i.e. the amplitude of the seasonal cycle) increases and decreases with a period of 21000 years. The temporal change in seasonality was incorporated in the function for the seasonality in incoming food in a simplified way by setting the precession cycle to $\beta_{\mathrm{p}}=1+\epsilon_{\mathrm{p}} \sin \left(2 \pi t / T_{\mathrm{p}}\right)$, with a period $T_{\mathrm{p}}$ of $21,000 \mathrm{yr}$. The amplitude $\epsilon$ of the seasonal cycle was set to $\epsilon=0.7$, to study specifically the evolutionary dynamics in the vicinity of the bifurcation point. The amplitude $\epsilon_{\mathrm{p}}$ of the precession cycle was set to a value smaller than one, $\epsilon_{\mathrm{p}}=0.25$, so that a certain amount of seasonality remained throughout the whole period. Initial body size is set close to its evolutionary equilibrium predicted by the (AD)-theory for $\epsilon_{\mathrm{p}}=0.0$.

\section{Results}

\subsection{Effects of seasonality}

Figure 1 shows the density fluctuations of food and rodents on an ecological (populationdynamical) time-scale, at which the body size is constant. Food $X_{0}$ (dotted-line) are transformed into rodent reserves $e_{1}$ (solid-line) and then into rodent biovolume $X_{1}$ (long dashedline). It can be seen that all variables follow the forced fluctuations of the incoming food density $X_{0, \text { in }}$ (short dashed-line), though each with an increased phase-shift and a decreased amplitude. This illustrates the buffering function of the reserves, smoothing out variations in the food availability. Muller and Nisbet (2000) studied the physiological response on an ecological time-scale, of organisms following DEB-rules in more detail. They showed that a variable food supply stimulates growth, increases mortality and may enhance reproduction.

Figure 2A shows how evolutionary equilibria of body size are related to food productivity $\bar{X}_{0, \text { in }}$, while $\epsilon=1$. Only at small productivities that are on average below the saturation constant, the body size at the evolutionary equilibrium decreases. At very small productivities 


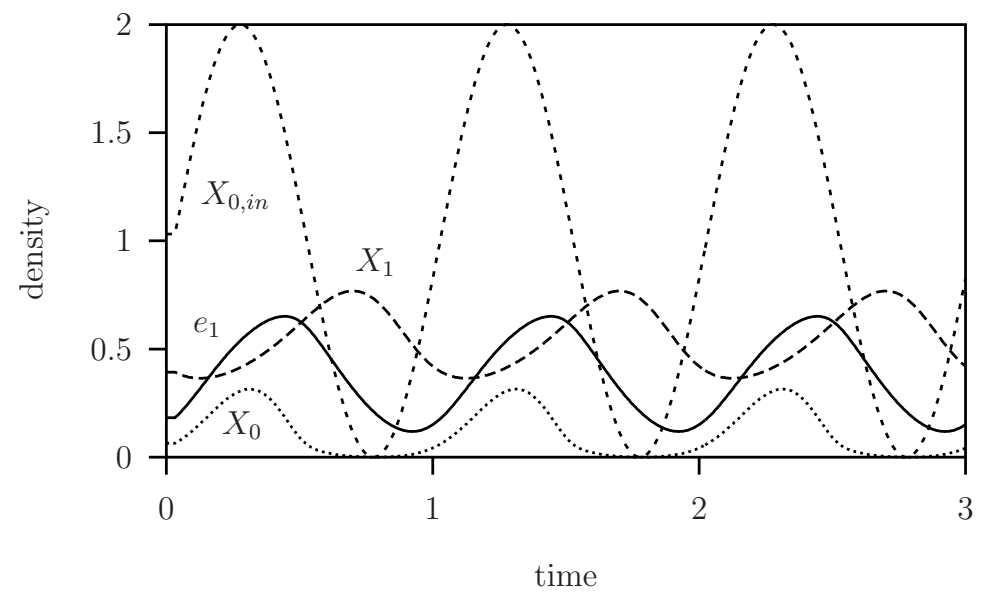

Figure 1: Periodical solution with $T_{0}=1 \mathrm{yr}$ at the ecological time-scale where $\bar{X}_{0, \text { in }}=100$ and $\epsilon=1$. Density fluctuations on an ecological time scale: Incoming food density $X_{0, \text { in }} / \bar{X}_{0, \text { in }}$ (short-dashed-line), food density $X_{0} / \bar{X}_{0, \text { in }}$ (dotted-line), rodent energy density $e_{1}$ (solid-line) and rodent biovolume density $X_{1} / \bar{X}_{0, \text { in }}$ (long-dashed-line) plotted against time (yr); energy density is a dimensionless variable, the other variables were normalized to the average incoming food density $\bar{X}_{0, \text { in }}=100$. The 'no starvation threshold' is passed when $e_{1}=\alpha=0.25$.

on the left-hand side of the 'tangent bifurcation', the population goes extinct.

Figure 2B shows the evolutionary equilibria of body size (solid and dashed-lines) against the amplitude $\epsilon$ of the environmental cycle; both period length and offset are kept constant. The average food productivity density equals $\bar{X}_{0, \text { in }}=100$. When the amplitude becomes larger than the offset, periods with zero food occur. The evolutionary equilibrium at the solid-line is stable, and thus an 'evolutionary attractor', which means that the population will evolve towards it. The dashed-line curve denotes an unstable equilibrium, or an 'evolutionary repeller': above the line evolution is directed towards larger body sizes, whereas below the line it is directed towards smaller body sizes.

The dotted-line curve denotes the 'no starvation threshold' body sizes. Above this threshold reproduction is never smaller than zero, such that the organisms always have sufficient energy reserves to pay their maintenance. Combining Eqns. (2.3) with (2.4) gives that the 'no starvation threshold' occurs at $e_{1}=\alpha=0.25$. Notice that at a point on this curve the population still goes extinct when the average reproduction rate $R$ over one period is smaller than the per capita mortality rate $h$ (see Eqn. (2.8)).

The figure shows that body size at the evolutionary equilibrium increases with the amplitude of the seasonal cycle. At $\epsilon=0.751$, the stable and unstable (solid and dashed lines) equilibria come together and the evolutionary equilibria 'disappear'; this is a so-called 'tangent bifurcation'. At this and smaller amplitudes, the environment will select for ever smaller body sizes. In reality the population goes extinct when the size comes below a threshold size below which the individuals cannot live. 


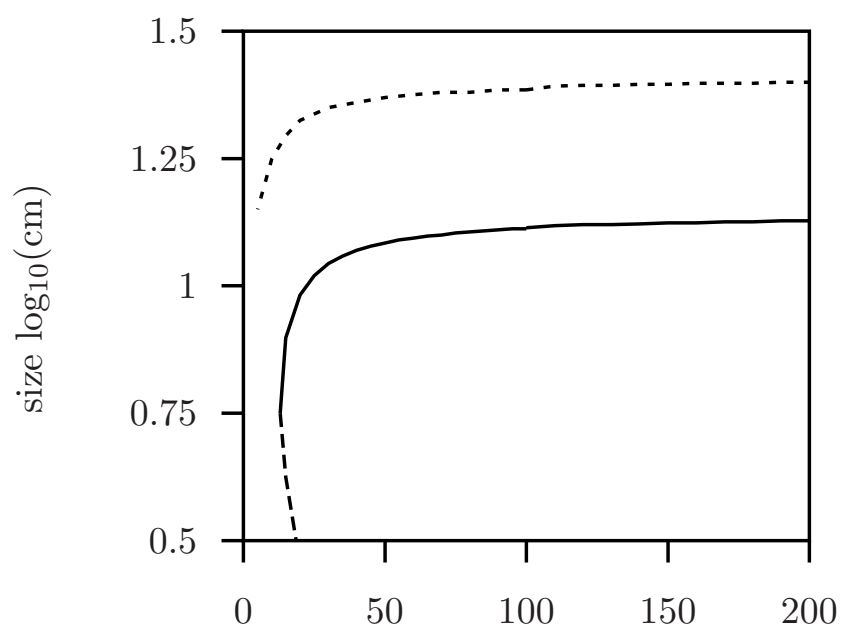

A

$$
\bar{X}_{0_{\text {in }}}
$$

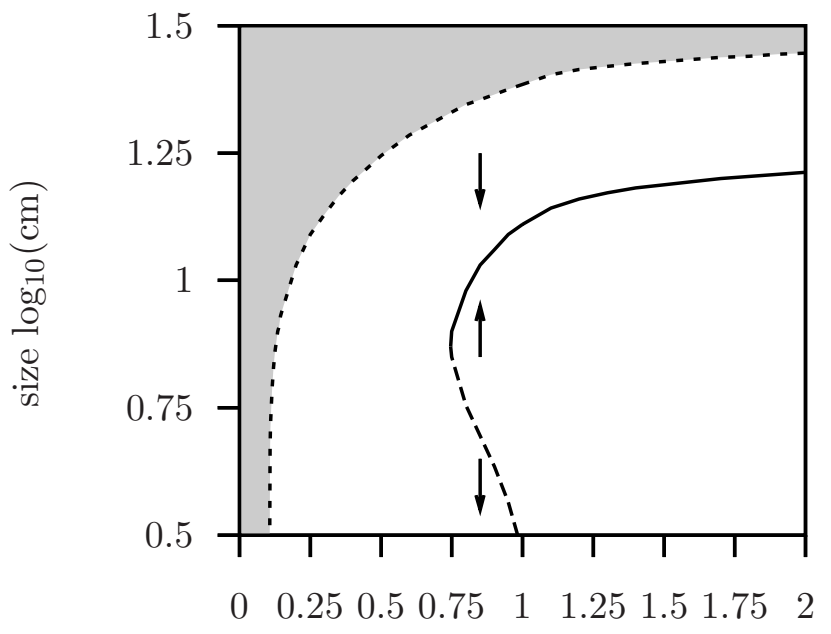

$\mathrm{B}$

$\epsilon$

Figure 2: The effects of (A) productivity, $\bar{X}_{0, \text { in }}$, and (B) seasonality, $\epsilon$, on the evolutionary equilibrium of body size. The shaded area indicates regions with 'no starvation threshold' where reproduction is always positive. The arrows indicate the direction of the evolution. The figure plots the trait $\log _{10}\left(\ell_{\mathrm{A}}\right)$, the logarithm of the body length in $\mathrm{cm}$, against the amplitude $\epsilon$ of the environmental cycle. The solid-line denotes the stable evolutionary equilibrium (attractor); the dashed-line curve denote the unstable evolutionary equilibrium (repeller); dotted-lines denote the 'no starvation threshold' body size. The stable evolutionary equilibrium at the reference values $\bar{X}_{0, \text { in }}=100$ and $\epsilon=1$ is shown in Figure 1. 

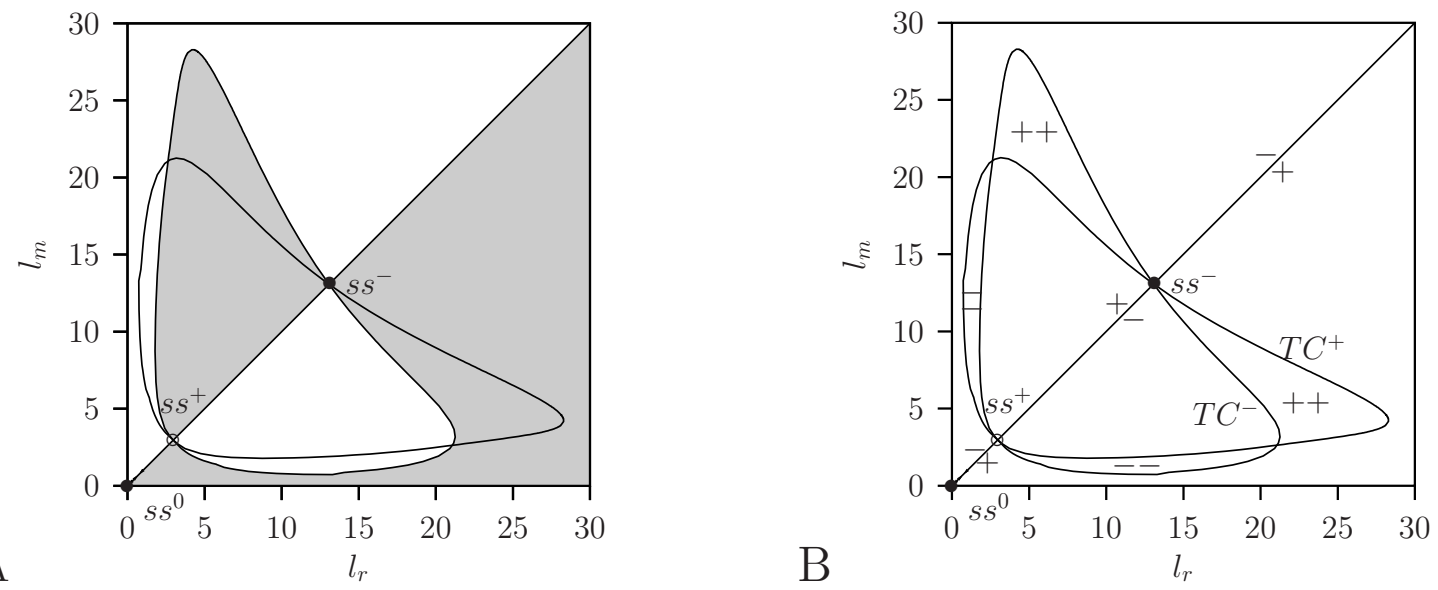

Figure 3: Pairwise Invasibility Plot (PIP) (A) and the Mutual Invasibility Plot (MIP) [12] (B). The shaded area indicates regions where the mutant's fitness, $s_{\text {mut }}$, is positive, that is where the mutant can invade the resident population. $\left(\ell_{r}, \ell_{m}\right)$ denote the length of the resident and mutant population that is the resident's and mutant's strategy, '+'-mutant may invade and replace the resident, '-' and '--'-mutant cannot invade, ' ++ '-mutant can invade but not replace the resident (mutual invasibility leading to coexistence). There are two singular strategy points $s s^{-}$and $s s^{+}$. The origin $s s^{0}$ is a population with individuals with zero length $\ell_{A}=0$, it is reachable and an evolutionary end-point. Parameter values: $\bar{X}_{0, \text { in }}=100, K=10$ and $\epsilon=1$ as in Figure 1 .

In order to study what happens in more detail we calculated so-called pairwise invasibility plots (PIP) and the Mutual Invasibility Plot (MIP) [12]. The PIP for $\epsilon=1$ is given in Figure 3left. At the diagonal the mutant equals the resident, which in this study means that they have the same body size. In the shaded regions the mutant population can invade the resident population. Point $s s^{-}$is evolutionary stable and convergence stable, and therefore a continuously-stable strategy [12, Figure 2 case (c)] and point $s s^{+}$is evolutionary unstable and convergence unstable similar to [12, Figure 2 case $(\mathrm{h})]$.

The MIP-plot Figure 3B can be obtained as the superposition of the PIP-plot and its mirror image along the principle diagonal [12]. Here it is calculated using a bifurcation analysis with $\left(\ell_{r}, \ell_{m}\right)$ as bifurcation parameters. The parameter space $\left(\ell_{r}, \ell_{m}\right)$ (length of the resident and mutant adults) is divided into four different regions: '+'-the mutant can invade and replaces the resident, '-'-the mutant cannot invade and goes extinct, '++'-the mutant can invade and both resident and mutant population can coexist but there is no protected dimorphism, '--'-the mutant cannot invade, since in this region both resident and mutant populations are stable. There regions are separated by transcritical bifurcations $T C^{+}$and $T C^{-}$. All regions except the latter case are described in [20].

The PIP plots for smaller $\epsilon$-values: $\epsilon=0.875, \epsilon=0.752, \epsilon=0.751, \epsilon=0.748$ are given in Figure 4.

With $\epsilon=0.875$ the plot shown in Figure 4A resembles the plot for $\epsilon=1$ shown in Figure 3. In Figure 4B point $s s^{-}$becomes evolutionary unstable. At this $\epsilon$-value, $\epsilon=0.752$, invasibility switches from [12, Figure 2 case (c)] into [12, Figure 2 case (b)]. Hence $s s^{-}$is still 

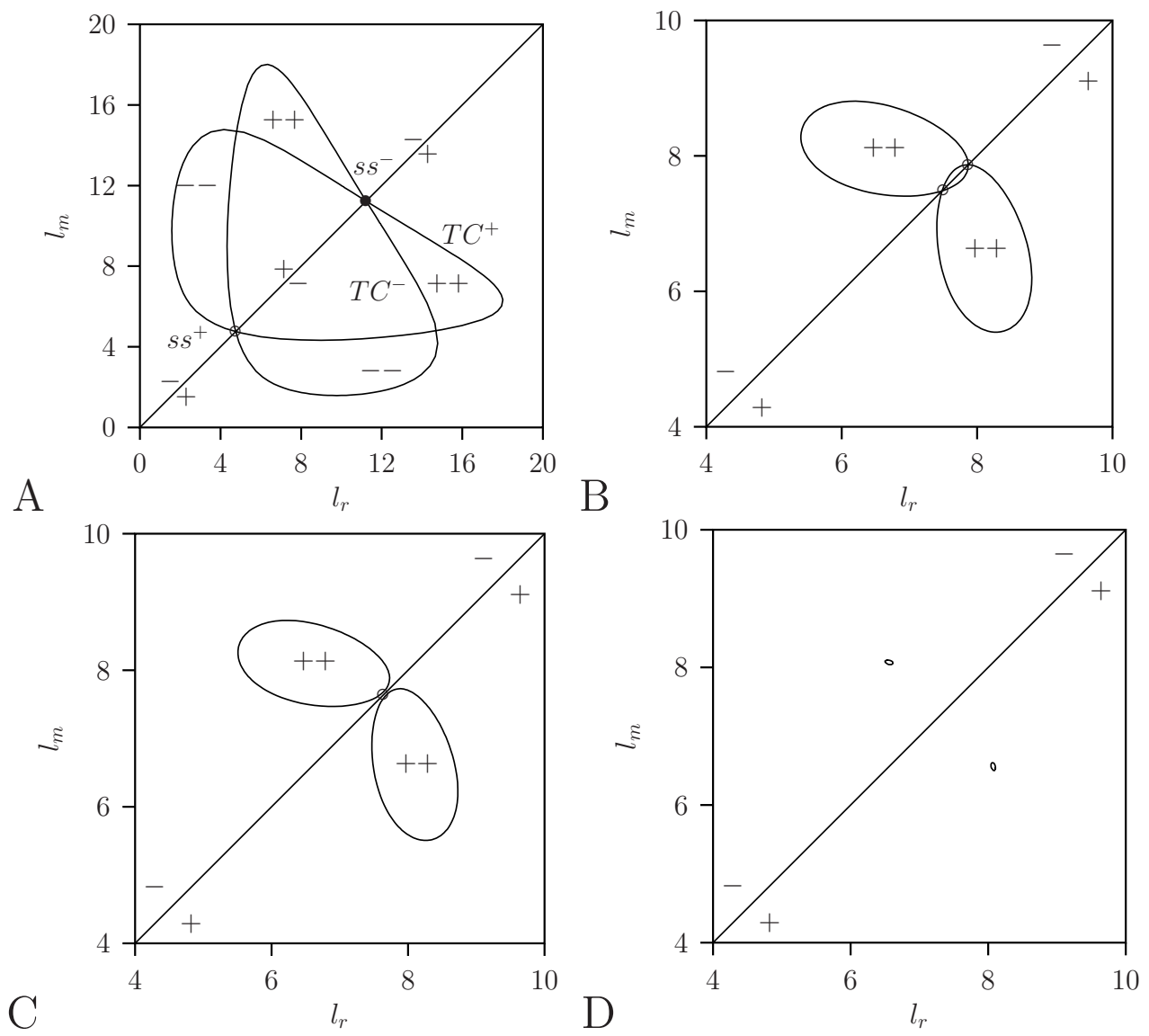

Figure 4: PiP-plots with $\epsilon=0.875, \epsilon=0.75171, \epsilon=0.75107$ and $\epsilon=0.748$ are shown. At point $\epsilon=0.75171 \mathrm{ss}^{-}$becomes evolutionary unstable. At $\epsilon=0.75107$ both $s s^{-}$and $s s^{+}$coincide. $\left(\ell_{r}, \ell_{m}\right)$ : length of the resident and mutant adults, '+'-mutant may invade and replace the resident, '-'mutant cannot invade, ' ++ ' mutant may invade but cannot replace the resident (mutual invasibility resulting in coexistence).

convergence stable but not evolutionary stable (in the figures indicated by an open circle). As a result, disruptive selection induces the difference in the trait values of the two coexisting strategies to grow. No protected dimorphism exists, however, all evolutionary paths leave the area of coexistence ' ++ ' via a bottom part of its boundary where a resident goes extinct. Evolution stops at the point $s s^{0}$ in the origin, which is an evolutionary end-point where the population will consist of individuals with zero length $\ell_{A}=0$.

For $\epsilon=\epsilon_{T}=0.751$, Figure $4 \mathrm{C}$, both singular evolutionary strategy points $s s^{-}$and $s s^{+}$coincide. When $\epsilon$ is lowered the region where both resident population and mutant population coexist does not cross the diagonal axis and are therefore not reachable via small mutation steps. Observe that on the ecological time-scale both populations can coexist but on the evolutionary time scale the singular strategy point is not stable and there is always extinction. At $\epsilon=0.748$, Figure $4 \mathrm{D}$, the regions where both resident and mutant population 
can coexist disappears. These patterns are similar to that found in [14, Figure 12] for a discrete time model and in [20, Figure 10] for a similar continuous time model. We refer the interested reader to [20] for a more detailed discussion of these results.

\subsection{Effects of variations in seasonality}

Results of the first simulation are shown in Figure 5, where the irregular curve is the evolutionary trajectory of the average body size and the regular curves denote the evolutionary equilibria.

Figure 5A shows the average body size trajectory, for seasonality $\epsilon=0.875$ and constant seasonality that is no precession cycle: $\epsilon_{\mathrm{p}}=0$. The vertical curve terminates close to the evolutionary singular strategy curve which was predicted by the (AD) theory (see also Figures 3 and $4 \mathrm{~A}$ ).

Figure 5B shows the trajectory for seasonality $\epsilon=0.875$ but now $\epsilon_{\mathrm{p}}=0.1$ and the initial trait value is set to the evolutionary equilibrium. Observe that the trajectory remains now rather close to the evolutionary singular strategy curve.

In Figure 5C, the amplitude of the seasonal cycle is $\epsilon=0.7$ and the variability of this seasonality is $\epsilon_{\mathrm{p}}=0.25$. As the starting point we set $\ell_{A}=0.9$. For seasonality $\epsilon=0.7$, and no precession cycle, $\epsilon_{\mathrm{p}}=0$, no positive evolutionary singular strategy exists (see Figure 2B). Here we find that also with a precession cycle $\epsilon_{\mathrm{p}}=0.25$ the population finally goes also extinct because the body size becomes zero.

In Figure 6 the same three simulations are plotted against time. These graphs also show the coexisting size class composition and how this changes throughout time. In all three cases the simulation is started with a single population at $\ell_{A}=\log _{10}(0.9)$, but new size classes arise due to mutations. These size classes temporarily coexist because they occur before others have had the time to go extinct.

In Figure 6A, there is no precession cycle $\epsilon_{\mathrm{p}}=0$. After $21 \mathrm{Kyr}$ the average body size is close to the evolutionary end-point predicted by the (AD)-theory, and the number of size classes stabilizes. The (AD)-theory predicts a single population for the stable evolutionary strategy due to competitive exclusion. In our simulations, however, many populations with different trait values coexist; the number of coexisting size classes depends on the chosen width of the size classes and on the variance of the mutation probability distribution. This coexistence is thus not due to mutual invasibility, but because mutations occur before an ecological equilibrium is reached. In Figure $6 \mathrm{~B}$, the average body size changes sinusoidallike due to the precession cycle $\epsilon_{\mathrm{p}}=0.1$. In Figure $6 \mathrm{C}$ the average body size changes again oscillatory but the mean is decreasing. The figure shows only the transient behaviour, namely only the first two cycles of the trajectory shown in Figure $5 \mathrm{C}$. When simulations are continued the population goes finally extinct. 

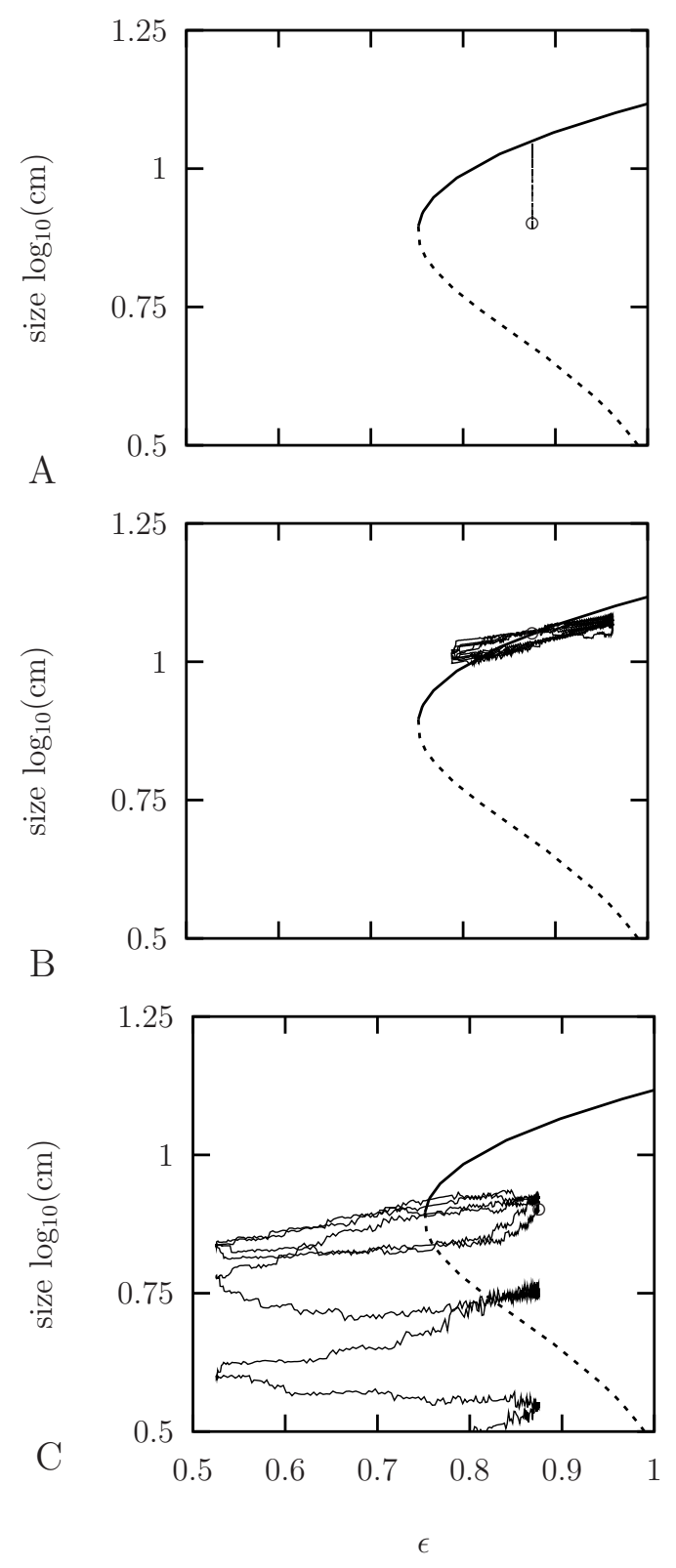

Figure 5: Three evolutionary trajectories of the average adult body sizes (irregular curve) plotted against seasonality whereby the seasonal amplitude is varied according to the changes in precession; the regular curves show the evolutionary equilibria. A. Simulation results for $\epsilon=0.875$ (vertical line) and $\epsilon_{\mathrm{p}}=0$, that is no. The initial trait value $\ell_{\mathrm{A}}=\log _{10}(0.9)$ is indicated by an open circle. B. Simulation results for $\epsilon=0.875$ and $\epsilon_{\mathrm{p}}=0.1$. The initial trait value is now the evolutionary singular strategy value predicted by the (AD)-theory for $\epsilon=0.875$ and $\epsilon_{\mathrm{p}}=0.0$. C. Results for an seasonal amplitude of $\epsilon=0.7$ and a temporal variability in seasonality of $\epsilon_{\mathrm{p}}=0.25$. Eventually the population goes extinct. See Figure 6 for the transient evolutionary dynamics. 


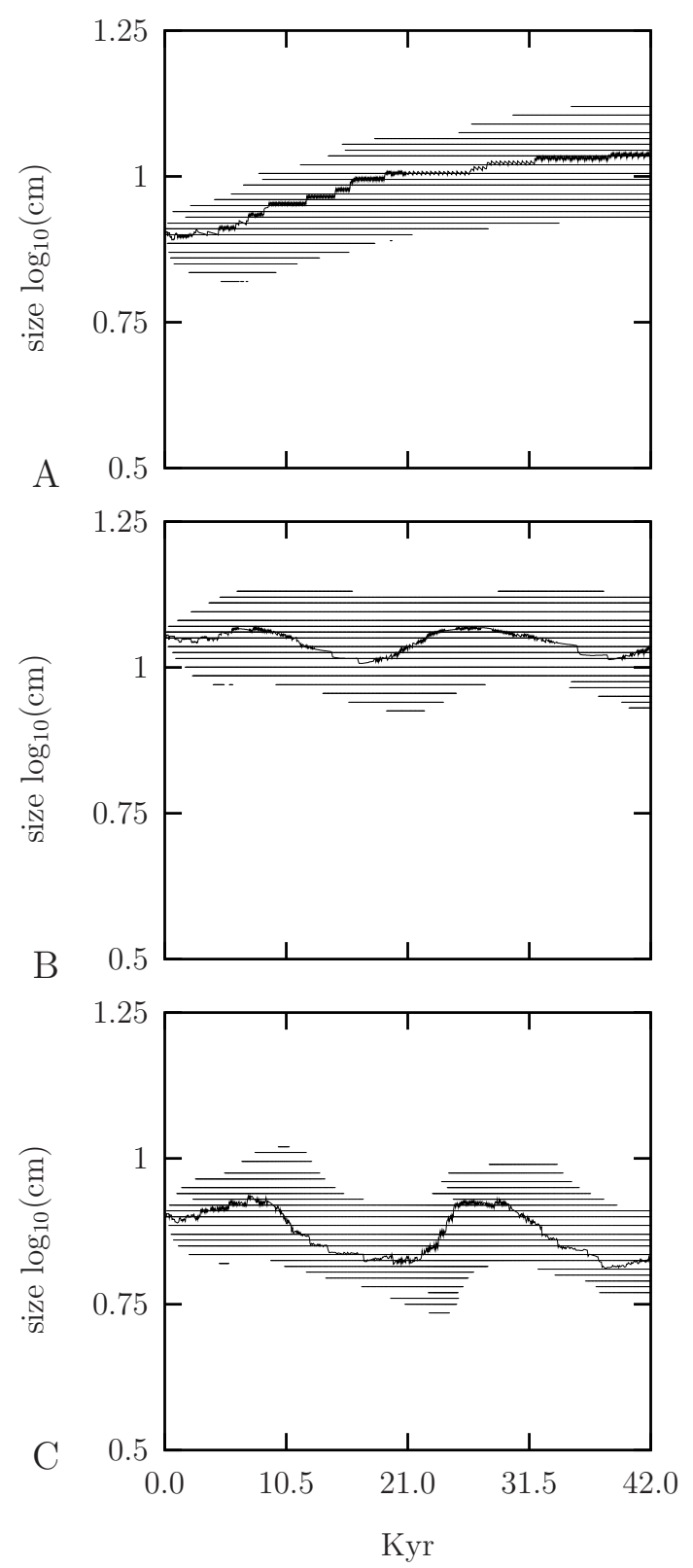

Figure 6: The (transient) evolutionary trajectory of the average adult body sizes and the distribution of the body size classes plotted against time. A. resulting from a simulation in which $\epsilon=0.875$ and $\epsilon_{\mathrm{p}}=0$. B. resulting from a simulation in which $\epsilon=0.875$ and $\epsilon_{\mathrm{p}}=0.1$. C. resulting from a simulation in which $\epsilon=0.7$ and $\epsilon_{\mathrm{p}}=0.25$. The horizontal lines indicate the time intervals where the size class is present in the system. Often multiple classes exist simultaneously (no time-scale separation). See Figure 5 for the evolutionary trajectories. 


\section{Discussion}

At the ecological time-scale, the dynamics of the population follow that of the available food source (see Figure 1). At the evolutionary time-scale, the model results show that (above a certain threshold of the amplitude of the environmental cycle) a positive relation exists between this amplitude and the body size at the evolutionary equilibrium (Figure 2B). As the amplitude corresponds to the variability and therefore to the seasonality of the environment, these results support the seasonality hypothesis of Lindstedt and Boyce (1985).

Our model results contrast most sharply with the model results of [38], who also used a process-based model but found that variability selects for smaller sizes. This contrast originates from the structural differences between the two models. In the model of Shertzer and Ellner (2002), energy storage is treated as an additional body feature that is only used in times of need. In contrast, in DEB theory the reserve dynamics are based on physiological rules: energy reserves are given a much more central role and are more closely linked to other physiological processes such as growth and reproduction. Assimilates derived from food are added to the reserves, which then fuel all other processes including maintenance, growth and reproduction. As a result, growth and reproduction do not depend directly on the available food, but on the reserves. Because of their central role, the energy reserves and their dynamics are fully embedded in the model. The assumptions underlying the reserve dynamics also lead to various body size scaling-relationships. As mentioned above, one of these is the scaling of storage capacity (maximum energy density $\left[E_{\mathrm{m}}\right]$ ) with body size, which relationship is supported by empirical data [36, 27]. In the model of Shertzer and Ellner (2002), however, the size of the energy storage is not physiologically limited, and energy capacity and body size evolve independently. The contrasting findings illustrate the importance of a physiologically based model structure.

Furthermore, Figure 2B shows that below a certain threshold in the amplitude of the environmental cycle, evolution is directed towards ever smaller body sizes. Apparently, if seasonality is sufficiently small, energy reserves are no longer needed, and the corresponding advantage of having a large size disappears. Obviously, the result that the modeled organisms will in that case evolve to a size of zero, is unrealistic. The reason that it occurs in the model is probably because some disadvantageous of small sizes were not taken into account. These may for instance be the vulnerability to predation, or the disability to digest certain types of food. These disadvantages, as well as various other complex processes and/or behaviours such as hoarding and torpor, were not included in the model, in order to single out the effects of seasonality on body size. The same holds for the adaptive change of allocation patterns $[35,33]$, which may lead to counter-intuitive results, such as why in some cases body size is not related to starvation time [17].

Similar to smaller seasonalities, also smaller environmental productivities may lead to smaller body sizes (Figure 2A). This relationship is also found in [38], and may be explained by the lower maintenance and reproduction costs of small individuals, as well as by their higher relative food intake rate. Also, it may be related to the density dependence of the fitness measure, as increased mortality during the bad season reduces competition during 
the good season.

In addition to the evolution of body size in response to environmental variability at an ecological time scale, we also studied the evolution of body size in response to environmental changes occurring at a geological time scale. The patterns resulting from the simulations show that, when variability in seasonality is included (Figure 5A and $\mathrm{C}$, and $6 \mathrm{~A}$ and $\mathrm{C}$ ), a oscillatory pattern of the body size evolution arises during at least two 21-Kyr precession cycles. Body size cycles on this temporal scale have been observed, for instance in a Last Glacial Maximum present day record based on the size of faecal pellets of bushy-tailed woodrats from the Great Basin and Colorado Plateau (US) [39]. The authors attributed the observed body size decline to warming, leading to a selection for higher surface to volume ratios (temporal interpretation of "Bergmann's Rule"). Our simulation illustrates that such evolutionary patterns may also result from changes in seasonality. Environmental periodicity and cyclical body size responses may exist on even longer timescales, which is studied in a planned paper on the Late Miocene fossil record of rodent teeth in the Teruel Basin in Central Spain.

As has been discussed, in case of seasonal cycles with a fixed amplitude $\epsilon$, the evolutionary steady-states can be calculated with using (AD)-theory. However, due to fluctuations in seasonality $\epsilon_{\mathrm{p}}>0$, some of the requirements for applying (AD)-theory may not be fulfilled (no time-scale separation) and therefore a stochastic simulation process was used. Indeed, it can be seen that new mutants are introduced already before the ecological system reaches an equilibrium, leading to coexistence of multiple body size classes (Figure 6). Yet, our simulations show that if the variability in seasonality is small (Figure 5A), and/or the seasonality is sufficiently far from the bifurcation point (Figure $5 \mathrm{~B}$ ), the average of the trait distribution is close to the evolutionary equilibrium trait value predicted by the (AD)-theory. An explanation for this is that in these cases the evolutionary equilibrium does not change very fast with time, and evolution can keep up with these changes.

\section{References}

[1] K. G. Ashton. Body size variation among mainland populations of the western rattlesnake (Crotalus viridis). Evolution, (2001), 55(12):2523-2533.

[2] M. S. Boyce. Climatic variability and body size variation in the muskrats (Ondatra zibethicus) of North America. Oecologia, 36 (1978), 1-19.

[3] M. S. Boyce. Seasonality and patterns of natural selection for life histories. The American Naturalist, 114 (1979), No. 4, 569-583.

[4] S. L. Chown, C. J. Klok. Altitudinal body size clines: latitudinal effects associated with changing seasonality. Ecography, 26 (2003), No. 4, 445-455.

[5] D. Cohen, H. Parnas. An optimal policy for the metabolism of storage materials in unicellular algae. J. theor. Biol., 56 (1976), 1-18. 
[6] C. R. Dickman, P. S. Mahon, P. Masters, D. F Gibson. Long-term dynamics of rodent populations in arid australia: the influence of rainfall. Wildlife Research, 26 (1999), No. 4, 389-403.

[7] U. Dieckmann. Can adaptive dynamics invade? Trends in Ecology and Evolution, 12 (1997), 128-131.

[8] U. Dieckmann, R. Law. The mathematical theory of coevolution: a derivation from stochastic processes. J. Math. Biol., 34 (1996), 579-612.

[9] R. L. Dunsbrack, M. A. Ramsay. The allometry of mammalian adaptations to seasonal environments - a critique of the fasting endurance hypothesis. OIKOS, 66 (1993), No. 2, 336-342.

[10] S. H. Ferguson. The effects of productivity and seasonality on life history: comparing age at maturity among moose (Alces alces) populations. Global Ecology and Biogeography, 11 (2002), No. 4, 303-312.

[11] N. R. French, D. M. Stoddart, B. Bobek. Patterns of demography in small mammal populations. In F. B. Golley, K. Petrusewicz, and L. Ryszkowski, editors, Small mammals: their productivity and population dynamics, pages 73-102. Cambridge University Press, Cambridge, 1975.

[12] S. A. H. Geritz, É. Kisdi, G. Meszéna, J. A. J. Metz. Evolutionarily singular strategies and the adaptive growth and branching of the evolutionary tree. Evolutionary Ecology, 12 (1998), 35-57.

[13] S. A. H. Geritz, J. A. J. Metz, É. Kisdi, G. Meszéna. Dynamics of adaptation and evolutionary branching. Physical Review Letters, 78 (1997), 2024-2027.

[14] S. A. H. Geritz, E. van der Meijden, J. A. J. Metz. Evolutionary dynamics of seed size and seedling competitive ability. Theoretical Population Biology, 55 (1999), 324-343.

[15] J. D. Hays, J. Imbrie, N. J. Shackleton. Variations in the earth's orbit: Pacemaker of the ice ages. Science, 194 (1976), 1121-1132.

[16] F. J. Hilgen, W. Krijgsman, C. G. Langereis, Lourens L. J., Santarelli A., W. J. Zachariasse. Extending the astronomical (polarity) time scale into the Miocene. Earth Planet. Sci. Lett., 136 (1995), No. 3, 496-510.

[17] K. L. Kirk. Life-history responses to variable environments: starvation and reproduction in planctonic rotifers. Ecology, (1997), 78(2):434-441.

[18] B. W. Kooi, S. A. L. M. Kooijman. Population dynamics of rotifers in chemostats. Nonlinear Analysis, Theory, Methods \& Applications, 30 (1997), No. 3, 1687-1698. 
[19] B. W. Kooi, S. A. L. M. Kooijman. Discrete event versus continuous approach to reproduction in structured population dynamics. Theoretical Population Biology, 56 (1999), No. 1, 91-105.

[20] B. W. Kooi, T. A. Troost. Advantages of storage in a fluctuating environment. Theoretical Population Biology, 70 (2006), No. 4, 527-541.

[21] S. A. L. M. Kooijman. Dynamic Energy and Mass Budgets in Biological Systems. Cambridge University Press, Cambridge, 2000.

[22] J. Laskar, P. Robutel, F. Joutel, M. Gastineau, A. C. M. Correia, B. Levrard. A longterm numerical solution for the insolation quantities of the earth. Astronomy \& Astrophysics, 428 (2004), 261-285.

[23] S. M. Lehman, M. Mayor, P. C. Wright. Ecogeographic size variations in sifakas: a test of the resource seasonality and resource quality hypotheses. American Journal of Physical Anthropology, 126 (2005), No. 3, 318-328.

[24] H. Lieth. Primary production: terrestrial ecosystems. Human Ecology, 1 (1973), 303332.

[25] M. Lima, J. E. Keymer, F. M. Jaksic. El Niño-Southern oscillation-driven rainfall variability and delayed density dependence cause rodent outbreaks in Western South America: linking demography and population dynamics. The American Naturalist, 153 (1999), No. $15,476-491$.

[26] C. C. Lindsey. Body sizes of poikilotherm vertebrates at different latitudes. Evolution, 20 (1966), 456-465.

[27] S. L. Lindstedt, M. S. Boyce. Seasonality, fasting endurance, and body size in mammals. The American Naturalist, 125 (1985), 873-878.

[28] T. Madson, R. Shine. Rainfall and rats: climatically-driven dynamics of a tropical rodent population. Austral Ecology, 24 (1999), No. 1, 80-89.

[29] S. Meiri, T. Dayan, D. Simberloff. Biogeographical patterns in the Western Palearctic: the fasting-endurance hypothesis and the status of Murphy's rule. Journal of Biogeography, 32 (2005), 369-375.

[30] J. A. J. Metz, S. A. H. Geritz, G. Meszéna, F. J. A. Jacobs, J. S. van Heerwaarden. Adaptive dynamics, a geometrical study of the consequences of nearly faithful reproduction. In: S. J. van Strien, S. M. Verduyn Lunel, editors, Stochastic and spatial structures of dynamical systems, pages 183-231. North-Holland, Amsterdam, 1996.

[31] J. A. J. Metz, S. A. H. Geritz, R. M. Nisbet. How should we define 'fitness' for general ecological scenarios? Trends in Ecology \& Evolution, 7 (1992), 198-202. 
[32] Milankovitch. Kanon der Erdbestrahlungen und seine Anwendung auf das Eiszeitenproblem. Royal Serbian Academy, Spec. Publ., 133 (1941), 1-633.

[33] E. B. Muller, R. M. Nisbet. Survival and production in variable resource environments. Bulletin of Mathematical Biology, 62 (2000), 1163-1189.

[34] P. E. Olsen, D. V. Kent. Long-period Milankovitch cycles from the Late Triassic and Early Jurassic of eastern North America and their implications for the calibration of the Early Mesozoic time-scale and the long-term behavior of the planets. Phil. Trans. R. Soc. Lond. (A), 357 (1999), 1761-1784.

[35] H. Parnas, D. Cohen. The optimal strategy for the metabolism of reserve materials in micro-organisms. J. theor. Biol., 56 (1976), No. 1, 19-55.

[36] R. H. Peters. The Ecological Implications of Body Size. Cambridge University Press, New York, 1983.

[37] M. Predavec. Population-dynamics and environmental changes during natural irruptions of Australian desert rodents. Wildlife Research, 21 (1994), No. 5, 569-582.

[38] K. W. Shertzer, S. P. Ellner. Energy storage and the evolution of population dynamics. J. theor. Biol., 215 (2002), 183-200.

[39] F. A. Smith, J. L. Betancourt, J. H. Brown. Evolution of body size in the woodrat over the past 25,000 years of climate change. Science, 270 (1995), 2012-2014.

[40] N. C. Stenseth, H. Leirs, A. Skonhoft, S. A. Davis, R. P. Pech, H. P. Andreassen, G. R. Singleton, M. Lima, R. S. Machang'u, R. H. Makundi, Z. B. Zhang, P. R. Brown, D. Z. Shi, X. R. Wan. Mice, rats and people: the bio-economics of agricultural rodent pests. Frontiers in Ecology and the Environment, 1 (2003), No. 7, 367-375.

[41] T. A. Troost, B. W. Kooi, U. Dieckmann. Joint evolution of predator body size and prey-size preference. Evolutionary Ecology, 22 (2008), 771-799.

[42] T. A. Troost, B. W. Kooi, S. A. L. M. Kooijman. Bifurcation analysis of ecological and evolutionary processes in ecosystems. Ecological Modelling, 204 (2007), No. 1/2, 253-268.

[43] I. M. M. van Leeuwen, F. D. L. Kelpin, S. A. L. M. Kooijman. A mathematical model that accounts for the effects of caloric restriction on body weight and longevity. Biogerontology, 3 (2002), No. 6, 373-381.

[44] J. D. Wigginton, F. S. Dobson. Environmental influences on geographic variation in body size of western bobcats. Canadian Journal of Zoology, 77 (1999), No. 5, 802-813. 